\title{
Effects of Nursing Intervention on Nursing Works in the Operation Room
}

\author{
Haiqin $\mathrm{Hu}^{*}$ \\ Hospital of Traditional Chinese Medicine of Wannian County, Shangrao, Jiangxi 335500, China
}

\begin{abstract}
Objective: To explore the effects of applying nursing intervention on nursing works in the operation room. Method: 60 patients who received surgeries in our hospital from mid-January 2013 to January 2014 were selected and then divided into the research group and the control group. Each group made up of 30 patients by random assignment. The research group was given operation room nursing intervention and the control group was given the routine operation room nursing. Then, the cooperation and anxiety of the two groups were compared accordingly. Result: After nursing, the research group obviously felt less anxious than the control group. There were significant differences between the two groups $(p<0.05)$; the operation room cooperation rate of the research group was significantly higher than that of the control group and significant differences can be found by comparing the two groups $(p<0.05)$. Conclusion: Giving nursing intervention to the surgery patients can effectively improve their cooperation and relieved their anxiety. Therefore, it is worth to be promoted widely in the clinical practice.
\end{abstract}

\section{KEYWORDS}

Nursing intervention

Operation room nursing

Anxiety

Cooperation

\section{Introduction}

In curing many clinical diseases, such as gastrointestinal cancer, uterine fibroids and so on, surgeries are necessary and almost all medical departments are involved in compulsory. No matter what kind of surgeries they are facing, the patients always feel anxious and panic. Their moods tend to change dramatically because the fear of the possible pain caused by the surgeries and worry about the success rate of the surgeries. This will affect the surgeries and the cooperativeness of the patients [1]. Therefore, it is necessary to provide the surgery patients with comprehensive nursing intervention in conjunction with the basis nursing conditions to improve the patients confidence in fighting the diseases. They can reduce their anxiety through psychological care, pain care, diet care, and etc., in order to make the patients be more cooperative with the medical staff. The authors selected 60 surgery patients who received treatment in their hospital from mid-January 2013 to

Copyright (C) 2016 Haiqin Hu

doi: $10.18686 /$ jn.v5i2.16

Received: June 24, 2016; Accepted: August 4, 2016; Published online: August 28, 2016

This is an open-access article distributed under the terms of the Creative Commons Attribution Unported License (http://creativecommons.org/ licenses/by-nc/4.0/), which permits unrestricted use, distribution, and reproduction in any medium, provided the original work is properly cited. ${ }^{\star}$ Corresponding author: Hospital of Traditional Chinese Medicine of Wannian County, Shangrao, Jiangxi 335500, China. E-mail: Huhaiqin11@126.com
January 2014 and gave 30 of them with operation room nursing intervention, which had achieved good nursing effect. The study of the author summed up as follows:

\section{Data and Methods \\ 2.1. General Data}

60 patients who received surgeries in our hospital from mid-January 2013 to January 2014 were selected and then divided into the research group and the control group. Each group made up of 30 patients by random assignment. In the research group, there were 17 male patients and 13 female patients with their ages ranging from 17 to 73 and their average age was $43.7 \pm 5.1$. Their Surgery types were as followed: 3 cases of pancreatic surgery, 7 cases of appendectomy, 4 cases of esophageal cancer surgery, 7 cases of orthopedic surgery, 6 cases of gastric carcinoma resection, 1 hemorrhoidectomy and 2 cases of myomectomy. In the control group, there were 18 male patients and 12 female patients with their ages ranging from 18 to 72 and average age was $43.5 \pm 5.5$. Surgery types were as followed: 4 cases of pancreatic surgery, 6 cases of appendectomy, 5 cases of esophageal cancer surgery, 6 cases of orthopedic surgery, 5 cases of gastric carcinoma resection, 2 hemorrhoidectomy and 2 cases of myomectomy. By comparing the general data of the two groups, there are no significant differences in terms of patient age, gender and surgery type, thus the two groups are comparable $(p>0.05)$. 


\subsection{Methods}

Control group: The group was given a routine operation room nursing, which mainly includes pre-operative hair removal, knowledge guidance and precautions.

Research group: In the conjunction with the basis of routine nursing, this group was given operation room nursing intervention. The specific steps were as below: First, psychological nursing: It was the first time for most patients to accept surgeries, so they were lacking understanding on operation knowledge. In this stage, their preoperative emotions were undulating and changeable, as they tend to suffer with fear, anxiety and other negative emotions. Thus, the circulating nurses must give them a detailed introduction and basic information on operation room, mainly including the placement of equipment, operating table position and the roles of related drugs, so that the patient will has a sense of familiarity and their psychological pressure can be relieved [2]. It was not allowed to directly address the patients by their bed numbers and they should be called affectionately by proper names, and by taking consider of their age and personality differences. Furthermore, communication with targeting patients should be strengthened by fulfilling and understanding the demands and concerns of patients in a dynamic manner. The patients should also be told about the safety and the importance of the surgery in order to help them build up confidence in the surgery. Second, communication strengthening: After completion of the surgery, daily discussions should be done about the conditions of the patients. Careful monitoring on changes of their illness should be done to find out whether there was any further pain and discomfort for them. At the same time, the nursing staffs were required to communicate more kindly with family members of the patients, so that the families of the patients can also encouraged the patients with care and warmth concern. Third, the pain nursing can help the patients who had to endure pain over a long period of time after the surgeries. Thus, the medical staff should rate the patients according to their degrees of pain and the pain can be divided into mild pain, moderate pain and severe pain. If the patients were in moderate or severe pain, the medical staff can provided them with oral pain medication or injecting pain medication. Fourth, the diet nursing should be strengthened and adverse food should be prohibited according to different types of surgery to prevent further complications. Specifically, the patients were asked to eat more food that rich in vitamins and proteins to supply for adequate nutrition in an all-around manner and to promote faster healing of the surgical wounds. Patients should not eat spicy and fried food to prevent discomfort on the body.

\subsection{Efficacy Judgment}

As for the experimental patients, Self-formulated Anxiety Assessment Scale was used accordingly. The higher the scores, the higher the anxiety levels of the patients. The anxiety assessment was conducted for 3 days, one assessment for one day before the surgery respectively. In the meantime, the cooperativeness degree of the patients was evaluated in the course of the operation.

\subsection{Statistical Analysis}

The clinical materials and data of the patients involved in this study were analyzed and handled with SPSS 18.0 Statistical Software. The quantitative data were handled with $\mathrm{t}$ test and the preoperative and postoperative data were handled with chi-squared test. It turns out that $p<0.05$, which means that there was significant difference and comparison results were statistically significant $[3,4]$.

\section{Results}

Comparison of the pre-nursing and post-nursing anxiety levels of the two groups of patients: Before the nursing, the anxiety scoring of the two groups of patients were $(40.50 \pm 6.91)$ points and $(40.61 \pm 7.18)$ points respectively, which show no significant difference and were not statistically significant $(p>$ $0.05)$; after the nursing, the anxiety scoring of the two groups of patients were respectively $(40.88 \pm 17.89)$ points and $(47.67$ \pm 8.48 ) with the anxiety level of the research group being obviously lower than that of the control group. The comparison showed a significant difference between the two groups $(p<$ 0.05). The specific data were shown in Table 1.

Table 1. Pre and post-nursing anxiety comparison of the two patient groups.

\begin{tabular}{cccc}
\hline Group & & Pre-nursing & Post-nursing \\
\hline Research group & 30 & $40.50 \pm 6.91$ & $40.88 \pm 17.89$ \\
Control group & 30 & $40.61 \pm 7.18$ & $47.67 \pm 8.48$ \\
Value of $\mathrm{P}$ & & $>0.05$ & $<0.05$ \\
\hline
\end{tabular}

Comparison of the cooperation degrees of the two groups of patients in the operation room: After the nursing, 16 patients of the research group were initiatively cooperative, 10 patients were ordinarily cooperative and 4 patients were reluctantly cooperative. In the control group, 10 patients were initiatively cooperative, 7 patients were ordinarily cooperative and 13 patients were reluctantly cooperative. It can be seen that the cooperation degree of the research group was obviously higher than that of the control group and there was significant difference in terms of cooperation degrees between the two groups $(p<0.05)$. The specific data were shown in Table 2.

\section{Discussion}

Surgery is the most effective treatment for most of the diseases and it is performed mainly by doctors using medical equipment by cutting off the patient's parts of body and then sewing up the incision wounds. The main surgical tools are knives, scissors and needles, etc. These tools are used to operate on the part of patients' bodies to maintain the health of patients. Surgery is the main treatment method adopted by the surgical department [5]. The goal of a surgery is to generally remove the diseased tissues, repair 
Table 2. Cooperation degree comparison of the two patient groups after nursing ( $n \%)$.

\begin{tabular}{ccccc}
\hline Group & $n$ & $\begin{array}{c}\text { Initiatively } \\
\text { cooperative }\end{array}$ & $\begin{array}{c}\text { Ordinarily } \\
\text { cooperative }\end{array}$ & $\begin{array}{c}\text { Reluctantly } \\
\text { cooperative }\end{array}$ \\
\hline $\begin{array}{c}\text { Research } \\
\text { group }\end{array}$ & 30 & $16(54.7)$ & $10(32.5)$ & $4(12.8)$ \\
$\begin{array}{c}\text { Control } \\
\text { group }\end{array}$ & 30 & $10(32.5)$ & $7(23.3)$ & $13(44.2)$ \\
$p$ value & $/$ & $<0.05$ & $<0.05$ & $<0.05$ \\
\hline
\end{tabular}

the damage, inhibit the trachea and improve the functions and shapes of the body. In surgery, the human body tissues need to be cut in open, therefore, it entails great trauma compared with other treatment methods. The scope of the surgeries keeps expanding and operations can be done on any part of the body with the development of the medical technologies. Moreover, the surgical instruments and facilities are updated with a new version in time from the initial scalpel, the stippling knife, the microwave knife, the ultrasonic knife to the laser knife, etc. Since surgery can causes damage to the human body, a variety of complications may occur after the surgery. For example, in case of gastrectomy, complications may occur such as cicatricle adhesions, vascular injury, coma, biliary fistula and pancreatitis, etc. after the surgery due to the urgency of this disease. After the surgery, the immunity capability of the patients decreases, which may cause a variety of infectious complications. For a long term, the operation causes changes of the channels and volume of the gastrointestinal tract, which may lead to fullness and bugling after meals, dizziness, nausea and other complications. The surgery will leave wounds of different sizes in different locations. The wounds can also be categorized into clean wounds and infected wounds. The clean wounds are basically achieved by using sterile equipment and can heal over certain of time through effective suturing. In the cleaning process, attention should be paid to protect the wounds from pathogenic bacteria to avoid infection. Usually stitches will be removed a week after the surgery. Debridement can be applied to the contaminated wounds, because they carry bacteria but are not already infected yet. The already infected wounds should be controlled and drugs should be applied to them on a regular basis to promote their healthy growth because they can induce acute inflammation due to the breeding and invasion of the bacteria. In case of healing by second intention, suture can be delayed timely [6].

Operation will do harm to both the body and the psychology of the patient. Most patients take a resisting attitude towards operations because of fear, and are worsened by medical malpractice. In particular, the mental state such as anxiety and insecurity of the clinical surgery patients are constantly changing all the time. In this experiment, the anxiety levels of both groups of patients change to different extents when approaching to the operations. However, the group accepting the nursing intervention obviously felt less anxious than the control group. The experimental results demonstrated that the implementation of reasonable nursing intervention can effectively relieve the patients' negative emotions such as fear and anxiety and help the rehabilitation of the patients. The reason why the patients tend to feel depressed, anxious and suffer with other abnormal emotions were because of due to lack of knowledge about the surgery and anesthesia, which would produce a negative impact on the success of the operation and the cooperation in operation. Anxiety is a common type emotional reaction. It is normal for the patients to feel painful due to the psychological threat of the operation and the individual's physiological balance can be broken through physiological nature mechanisms. In such case, the nursing intervention given to the patients can help in achieving good effect compared to drug treatment [7].

In this experiment, the patients of the research group were given operating room nursing intervention, which had achieved a good nursing effect. After nursing, the anxiety level of the research group was obviously lower than that of the control group, and the patients of the former group were obviously more cooperative in the operation room. The comparison had found out that there were significant difference between the two groups $(p<0.05)$. These showed that implementing operation room nursing intervention on the surgery patients can effectively improve the cooperation rate of the patients, relieve anxiety and is worthy to be promote in clinical practice. The operating room nurses should keep improving their overall quality and professional competence in the nursing practice, by making efforts to study psychology knowledge, improving their skills of reassuring the patients and communication skills as well as pursing selfdevelopment in a comprehensive manner.

\section{References}

1. Qin J. Analysis of Applying Psychological Nursing Intervention in the Operation Room Nursing. Chinese Manipulation \& Rehabilitation Medicine. 2011;2(8):50-50.

2. Zheng $Y$, Luo M, Lei D, et al. Effectiveness Analysis of Applying Nursing Intervention in the Operation Room Nursing. Health Required. 2012;11(7):445-445.

3. Tao J. Effectiveness Analysis of Applying Nursing Intervention in the Operation Room Nursing. Health Required. 2011;6(11):302-304.

4. Zhao Y. Analysis of the Nursing Intervention Importance in Case of Operation Room Nursing Errors. Chinese Manipulation \& Rehabilitation Medicine. 2011;2(12):159-159.

5. Meng Q. Effectiveness Analysis of Applying Nursing Intervention in the Operation Room Nursing. Guide of China Medicine. 2013;15(27):716-717.

6. Huang F. Effectiveness Analysis of Comfort Care Used in Operating Room Nursing. China Health Industry. 2011;24(24):14-15.

7. Sun L, Zhao Y, Liu F. Effectiveness Analysis of Applying Nursing Intervention Strategy in the Operation Room. Guide of China Medicine. 2013;36(21):266-267. 